\title{
Influence of nanoparticles in PZT ferroelectric material properties and their applications to memory devices
}

\author{
Kurapati Srinivas \\ Nanotechnology Group, Department of Physics, GMR Institute of Technology, A.P. India \\ Email address: \\ srinkura@gmail.com, srinivas.k@gmrit.org
}

To cite this article:

Kurapati Srinivas. Influence of Nanoparticles in PZT Ferroelectric Material Properties and Their Applications to Memory Devices. American Journal of Nanoscience and Nanotechnology. Vol. 2, No. 3, 2014, pp. 56-62. doi: 10.11648/j.nano.20140203.15

\begin{abstract}
Ferroelctrics are technologically important materials, particularly for their applications in ferroelectric random access memory, based on semiconductor integrated technology have been a great success. Furthermore, these RAMS are very sensitive to radiation and this is detrimental for military and space applications. The properties of the layered perovskite ferroelectrics can be enhanced by addition or substitution of alternative cations. Among the important ferroelectric materials lead zirconate titanate (PZT), which is part of the solid solution formed between ferroelectric lead titanate and anti-ferroelectric lead zirconate with different compositions are used for different applications. Recent works indicate the influence of nanoparticles in PZT properties like decrease of synthesizing temperature, electrical conductivity and low dielectric loss. These materials will be the future ferroelectric materials for noval applications. The present paper Include a thorough study of these materials to finding out reasons for the improvement ferroelectric material properties in the nano scale and their optimization techniques for better applications.
\end{abstract}

Keywords: Ferroelectrics, Nanoparticles, Lead Zirconate Titanate, FeRAMS, SPM

\section{Introduction}

Ferroelectric materials exhibit a wide spectrum of functional properties, including switchable polarization, piezoelectricity, high non-linear optical activity, pyroelectricity, and non-linear dielectric behaviour. These properties are indispensable for application in electronic devices such as sensors, microactuators, infrared detectors, microwave phase filters and, ultimately, non-volatile memories[1-9]. Due to this unique combination of properties researchers and engineers have been focusing on ferroelectric materials for a long time. A number of classical monographs served as a comprehensive introduction into the field for several generations of scientists. However, recent advances in the synthesis and fabrication of micro- and nanoscale ferroelectric structures brought to life new physical phenomena that need to be studied and understood at this size range.

As structure dimensions are getting smaller, ferroelectric materials exhibit a pronounced size effect manifesting itself in a significant deviation of the properties of low-dimensional structures from the bulk properties. In this sense, ferroelectrics are similar to magnetic materials since surface energy cannot be neglected in small volumes and long-range dipole interaction is significantly modified in confined geometries. However, the intrinsic size effect in ferroelectrics is considerably weaker than in ferromagnetics. This can be simply understood from a comparison of domain walls in ferroelectrics (generally several unit cells wide with much thicker Bloch domain walls in ferromagnetics. This difference is due to the fact that exchange interactions between spins are much stronger than the dipole-dipole interactions in ferroelectrics. It also depends on whether a ferroelectric is confined in one-, two-, or all three-dimensional structures. As early as 1944, Onsager was able to show that in the two-dimensional lattice described by the Ising model a monolayer of non-oriented dipoles became oriented as a result of the 1st order phase transition. Many years later, rigorous experimental studies confirmed the conclusion that ferroelectricity might still exist in ferroelectric films with the thickness equal to just several unit cells.

To understand the scaling behaviour, several fundamental issues need to be addressed, including the intrinsic limit for a stable domain, extrinsic effects on polarization stability, mechanism of domain wall motion and switching in nanoscale volumes. This review paper summarizes recent advances in the quickly developing 
field of nanoscale studies of ferroelectrics, analyses its current status and considers potential future developments.

The paper begins with a brief overview of the fabrication methods of ferroelectric nanostructures, such as ultrathin films, nanocrystals, nanotubes and nanowires particularly PLZT materials and investigation of the size effects and stability of the ferroelectric phase in low-dimensional structures. Lead zirconate titanate nanoislands were obtained by a self-patterning method making use of the instability of ultrathin films during high-temperature treatments. After high-temperature annealing, the as-deposited film breaks into islands with a narrow size distribution. The crystallization temperature plays an important role in the morphology of the final structures Application of novel characterization techniques, notably scanning probe microscopy (SPM), played a critical role in the recent advances in science and technology of nanoscale ferroelectrics]. The paper summarizes the SPM-based methods applied for high-resolution characterization of ferroelectrics. One of the focuses of the review is the kinetics of nanoscale ferroelectric switching in inhomogeneous electrical and elastic fields probed by piezoresponse force microscopy (PFM). Recent insights into the mechanisms of ferroelectric fatigue and imprint made possible by the use of PFM are described as well. Another emphasis is made on tailoring the electrical and mechanical properties of ferroelectrics with a viewpoint of fabrication of nanoscale domain structures that can be used both for fundamental studies and for application related purposes. Scaling of the device dimensions to the range where ferroelectric materials start to show apronounced size effect has emphasized the importance of nanoscale studies of ferroelectric properties. The size effect in ferroelectrics manifests itself with the reduction in the sample geometrical dimensions by a decrease in the remnant polarization, dielectric permittivity and phase transition temperature, increase in the coercive field, changes in the domain structure, etc.

Device miniaturization poses not only technological and manufacturing challenges, it also requires the understanding of the phenomena occurring at nanoscale in condensed matter and at the involved interfaces. Ferroelectric (FE) materials, such as the renowned perovskites_BaTiO3, $\mathrm{PbZr} x \mathrm{Ti} 1-x \mathrm{O} 3, \mathrm{BiFeO} 3$ and layered perovskites_SrBi2Ta2O9_Bi,La_4Ti3O12_, have been proposed for nonvolatile ferroelectric random access memories since two decades. Ferroelectric perovskites can hold information in the form of the polarization direction of individual FE domains, because they usually have two thermodynamically equivalent ground states of opposite ionic polarization. Application of a positive or negative dc bias to the tip can induce $180^{\circ}$ polarization switching, orienting polarization upwards or downwards. Thus created domains can be imaged using SPM, which, therefore, provides both 'write' and 'read-out' capabilities. Ferroelectric materials have attracted wide attention due to their applications in nonvolatile memory devices (NVMDs) rendered by the electrically switchable spontaneous polarization. We summarize the recent development of the nonvolatile ferroelectric field effect transistor (FeFET) memory devices based on nanostructures. Future prospects of NVMDs.

\section{Different Types of Ferroelectric Nanostructure and Their Preparation}

Ferroelectric PZT thin films were prepared on $\mathrm{Pt} / \mathrm{Ti} /$ $\mathrm{SiO} 2 / \mathrm{Si}$ substrates by the sol-gel method. The raw materials were lead acetate rehydrate [Pb(OCOCH3)2.3H2O, 99.5\%], zirconium tetra n-propoxide $(\mathrm{Zr}(\mathrm{OC} 3 \mathrm{H} 8) 4,70 \%)$, titanium (IV) butoxide (Ti(OC4H9)4, 98\%) as precursor material and Methanol/Acetic acid mixed solvent as a solvent. Figure 1a illustrates the embossing process of the PZT film. After spin-on, the precursor film was first baked on hotplate at $60^{\circ} \mathrm{C}$ in air for $5 \mathrm{~min}$. Then, an embossing process was carried out at room temperature under a pressure of $9 \mathrm{Mpa}$ for 15 min using a silicon template, which has a grating $500 \mathrm{~nm}$ lines/spaces. The template used was first coated with an anti-stick layer on its surface in order to reduce its adhesion to the embossed gels and make it easier in later de-mold procedure. After embossing, the gel layers were first pyrolyzed in air on a hotplate at $350^{\circ} \mathrm{C}$ for $5 \mathrm{~min}$ and then crystallized by conventional thermal annealing in air at $650^{\circ} \mathrm{C}$ for $15 \mathrm{~min}$. Equally important for understanding the scaling behaviour of ferroelectrics was the significant progress in the development of material processing techniques that allowed fabrication of nanoscale ferroelectric structures, such as ultrathin epitaxial films, nanoscale capacitors, nanotubes and nanorods. A number of top-down and bottom-up approaches have been tried and tested, including electron-beam (EB) assisted fabrication, nanoprinting and self-assembling. The top-down methods that are widely used in modern nanotechnology provide high-precision positioning and size control but are limited in resolution. EB-assisted fabrication involves patterning the ferroelectric structures either by milling of the ferroelectric layer using a focused ion beam (FIB) or by maskless EB lithography. In the FIB patterning, introduced by Stanishevsky et al, nanoscale ferroelectric capacitors have been produced by 'cutting out' an isolated island from a Pt-coated Nb-doped $\mathrm{Pb}(\mathrm{Zr}, \mathrm{Ti}) \mathrm{O} 3$ (PZT). By optimizing the process it was possible to fabricate capacitors with lateral dimensions as small as $70 \times 70 \mathrm{~nm}^{2}$. The ferroelectric properties of as-processed capacitors which showed very weak hysteresis behaviour right after processing apparently due to the Ga damage could be recovered by annealing at $600{ }^{\circ} \mathrm{C}$. Still, the presence of a permanently damaged $10 \mathrm{~nm}$ thick layer has been reported.

A classical top-down patterning processing starts with a thin film, fabricating structures of desired geometry out of it by photolithography and etching. There are obvious advantages of this process, one being the starting "material" itself, which can be a well characterized thin 
film of high structural quality, along with the possibility of large area processing and controlled size and positioning. A broad spectrum of ultrathin ferroelectric films and nanodots were fabricated by various methods and thoroughly characterized, with emphasis on reducing the size below $100 \mathrm{~nm}$ while maintaining good ferroelectric properties. (Figure 1)Structural defects and ferroelectric domain patterns were investigated in order to have a good understanding of their influence on nanosized ferroelectrics and to figure out how to avoid their harmful effects. Epitaxial PZT 20/80 films were grown under optimized PLD conditions in order to achieve single crystallike FE thin films with very low density of extended structural defects. These high quality PZT films enabled fundamental studies of the electrical and ferroelectric properties, with minimized contribution from structural defects. Epitaxial FE nanodots[2] of various materials were successfully fabricated and geometrical ordering was achieved, allowing to approach the desirable memory density of Tbit/ in. 2 for potential application in nonvolatile ferroelectric data storage.

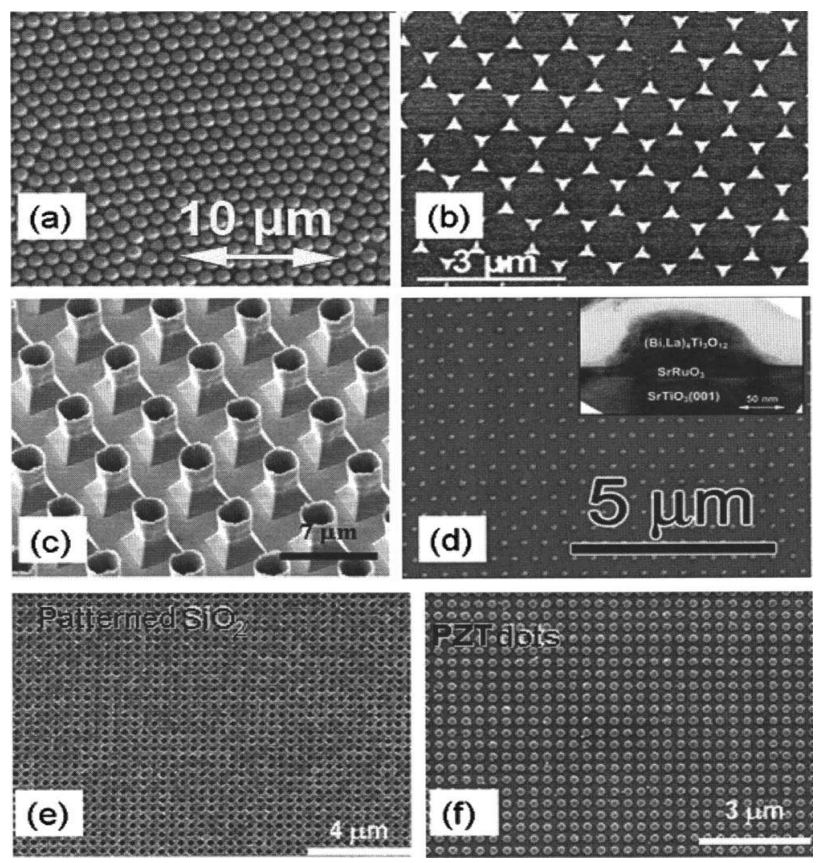

Figure 1. FE nanodots(NDs) produced by PLD: SEM micrographs of (a) a self-assembled monolayer of latex spheres used as mask for (b) NDs ofSrBi2Ta2O9,(c) gold nanotube membrane used as stencil mask for (d) Bi,La_4Ti3O12 NDs inset: cross section TEM of one of the dots(e)wafer-scale SiO2 membrane used for(f) PZT NDs.[2]

Recent advances in science and technology of ferroelectrics have resulted in the development of ferroelectric structures and devices with the length scales of several hundred nanometer and A and less. Typical device examples include, but are not limited to, thin films for nonvolatile ferroelectric random access memories (FeRAM), microactuators, microwave phase shifters and infrared sensors. Ferroelectrics are also considered as candidate gate-dielectric materials for field effect transistor applications due to their high permittivity. Scaling of the device dimensions to the range where ferroelectric materials start to show a pronounced size effect has emphasized the importance of nanoscale studies of ferroelectric properties

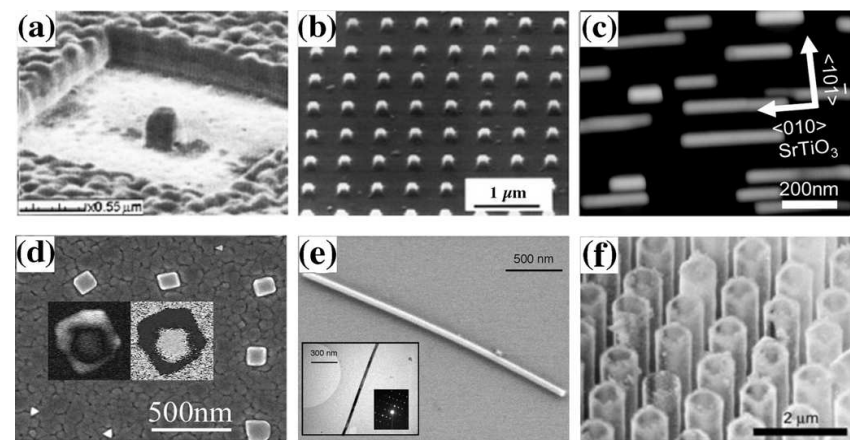

Figure 2. (a) Scanning ion beam image of the nanoscale PZT capacitor fabricated by FIB milling; (b)SEMimage showing a PZT cell array (c)AFM topographic image of self-assembled PbTiO3 nanoislands on the Pt/SrTiO3(101) substrate; (d) PZT(100)crystallites grown on 150 wideTiO2 seeds; (e)SEM image of an isolatedBaTiO3 nanowire; $(f)$ a micrograph of a free-standing array of SBT tubes with diameter of about $800 \mathrm{~nm}$ (Imagecourtesy of F Morrison, University of Cambridge)[3]

Lead zirconate titanate nanoislands were obtained by a self-patterning method making use of the instability of ultrathin films during high-temperature treatments. After high-temperature annealing, the as-deposited film breaks into islands with a narrow size distribution. The crystallization temperature plays an important role in the morphology of the final structures. The films annealed at higher temperature result in islands with pyramidal shape and increased height. For instance, the height of nanocrystals obtained from the 1:40 diluted precursor increases from $9 \mathrm{~nm}$ after a treatment at $800{ }^{\circ} \mathrm{C}$ up to 20 $\mathrm{nm}$ after a $1100{ }^{\circ} \mathrm{C}$ annealing. The area density decreases drastically down to $15-20$ islands per $1 \mathrm{~mm} 2$. We assume that at higher temperature the mobility and surface diffusion are enhanced and this allows the deposited material to migrate and to coalesce into larger islands. The ferroelectric properties of individual islands were probed by piezoresponse force microscopy.[6-9]. Islands with heights above $25 \mathrm{~nm}$ or a large lateral size show a well developed piezoelectric hysteresis loop. Experiments suggest that the ferroelectric properties are related rather to the volume than to the lateral size of the structures.

$\mathrm{Pb}(\mathrm{Zr}, \mathrm{Ti}) \mathrm{O} 3$ nanocrystals with different morphologies have been prepared hydrothermally with poly(vinyl alcohol) (PVA) and poly(acrylic acid) (PAA) as surfactants[6]. (Figure 3)The morphology of $\mathrm{Pb}(\mathrm{Zr}, \mathrm{Ti}) \mathrm{O} 3$ nanocrystals develops from a particle to a rod and then a wire by adjusting the ratio of PVA/PAA and reaction time in a hydrothermal system. The mixed surfactant system has been shown to promote anisotropic growth effectively and the formation of nanorods and single crystalline nanowires of $\mathrm{Pb}(\mathrm{Zr}, \mathrm{Ti}) \mathrm{O} 3$. A possible mechanism for such a shape evolution of nanocrystals is simply discussed However, achievement of shape control or shape-selective 
synthesis of ferroelectric oxide nanostructures by a simple method remains a challenge. Difficulty arises from the relatively complex crystal structure and rigid crystalline conditions of these oxidesPZT to test this possibility using poly(vinly alcohol) (PVA)/poly(acrylic acid) (PAA) as a surfactant system under hydrothermal conditions. Such an experimental design is based on the fact that PZT is highly attractive for both experimental and technological applications.

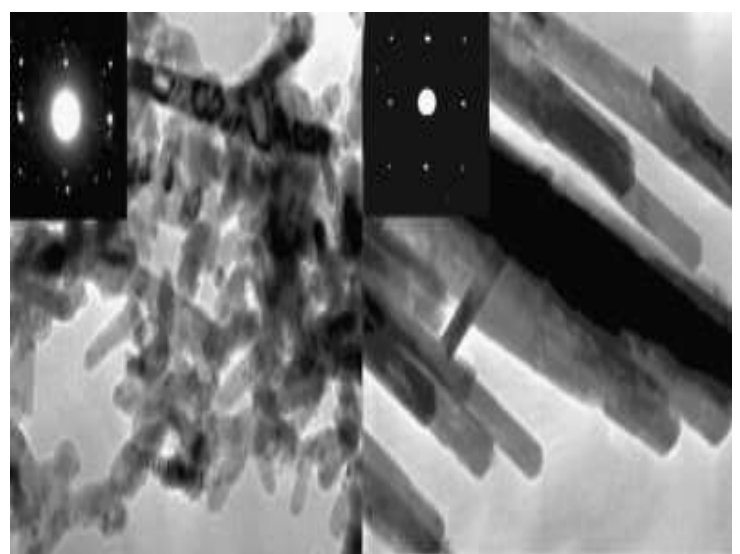

Figure 3. Transmission electron spectroscope (TEM) images of $\mathrm{Pb}(\mathrm{Zr}, \mathrm{Ti}) \mathrm{O} 3$ nanocrystals under hydrothermal conditions at $2001 \mathrm{C}$ with different poly vinyl alcoholpoly acrylic acid (PVA/PAA). (a) PVA/PAA51:3 (b) PVA/PAA51:6. The insets in TEM images show SAED patterns [6]

Scaling of the device dimensions to the range where ferroelectric materials start to show a pronounced size effect has emphasized the importance of nanoscale studies of ferroelectric properties. The size effect in ferroelectrics manifests itself with the reduction in the sample geometrical dimensions by a decrease in the remanent polarization, dielectric permittivity and phase transition temperature, increase in the coercive field, changes in the domain structure, etc.

\section{Scanning Probe Microscopy Techniques for Nanoscale Characterization of Ferroelectric Structures}

SPM techniques have revolutionized the field of ferroelectricity, for the first time providing an opportunity for non-destructive visualization of domain structures in ferroelectric thin films and testing the nanoscale ferroelectric structures.

SPM made possible nanoscale mapping of the surface potential, evaluation of local electromechanical properties, measurements of non-linear optical and dielectric constants. SPM has also opened new venues in nanoscale domain patterning for such applications as high-density data storage and ferroelectric lithography. Research groups in US, Europe and Asia are actively using SPM for high-resolution characterization of ferroelectric thin films, bulk ceramics, single crystals as well as for testing the scaling behaviour of ferroelectric nanostructures.

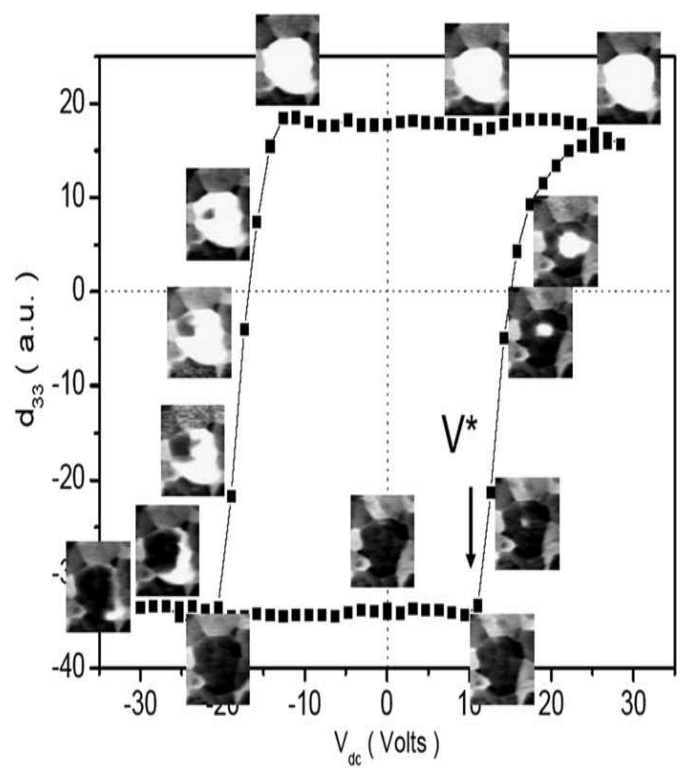

Figure 4. Local PFM hysteresis loop measured in a thick polycrystalline PZT film prepared by sol-gel. The insets (scan size 1 $1 \mu$ ) show the static domain structures obtained by scanning with ac voltage of amplitude $1 \mathrm{~V}$ in the corresponding points of the hysteresis .

An example of the visualization of the piezoelectric hysteresis in polycrystalline PZT films prepared by the sol-gel route is shown in figure 4. A sudden contrast change (arrow at $V *$ in figure 4) corresponds to the appearance of the stable inverse domain.

Thus, nucleation voltage can be considered as an analog of the coercive voltage in local measurements rather than the voltage where $d 33=0$. A rapid increase in $d 33$ with increasing $V \mathrm{dc}$ is explained by the forward and lateral growth of the nucleated domain, progressively contributing to the PFM signal. When the size of the inverse domain is much greater than the penetration depth of the weak ac field, the tip senses only a fully polarized area with aligned polarization and $d 33$ is well saturated. Figure 4 also illustrates the propagation of domain wall in polycrystalline ferroelectrics where local inhomogeneity and stresses make the Nanoscale ferroelectrics: processing.

Depending on the type of the interaction force between the probing tip and a sample - attracting or repelling - the SPM can operate in two different regimes: non-contact or contact, respectively. In the non-contact regime, the tip is scanned over the surface at a distance of 10-100 nm, which is controlled, for example, by monitoring the resonant frequency of the cantilever. The tip-sample interaction in this regime is dominated by the long range polarization and electrostatic forces. Because of this feature, non-contact SPM can be used for ferroelectric domain imaging by detecting the electrostatic field of the surface polarization charges. This mode of SPM, called electrostatic force microscopy (EFM)], has been predominantly applied to domain imaging in ferroelectric crystals or in thin films 
with micrometre-sized grains as the lateral resolution of this technique in ambient environment is relatively low $(\sim 40 \mathrm{~nm})$. Quantitative information on local surface potential related to spontaneous polarization can be obtained by means of scanning surface potential microscopy (SSPM), or Kelvin probe force microscopy (KPFM). This technique provides important information on the surface electronic properties of ferroelectrics, such as distribution of polarization and screening charges and their evolution during phase transitions. Application of KPFM is essential for determining the factors that govern the stability of the ferroelectric domains and control surface electrochemical reactivity .

Scanning capacitance microscopy (SCM) can be used for generating an image of trapped charges by measuring local tip-sample capacitance. SCM is not necessarily a non-contact mode of operation as the probing tip can be placed in direct contact with the sample surface to measure the sample capacitance. SCMcan be considered as a complementary approach to KPFM in the sense that it allows the estimation of the carrier concentration in the sub-surface regions of ferroelectric samples and the evaluation of the effect of the free carriers on polarization screening].

Dynamic methods, which include scanning non-linear dielectric microscopy (SNDM) and piezoresponse force microscopy (PFM) are based on detection of the electrical and mechanical response, respectively, of the sample to an ac voltage applied via a conductive probing tip.

Another dynamic contact SPM method is atomic force acoustic microscopy (AFAM) based on the detection of the cantilever displacement due to the mechanical excitation of the sample by an ultrasonic transducer which emits longitudinal waves into the sample and causes a sample surface displacement In the SNDM method, which employs a special resonator probe operating in the microwave frequency range, point-to-point detection of the local voltage-induced change in the sample capacitance allows nanoscale domain mapping in the surface layer of about $10 \mathrm{~nm}$ thick. It has been suggested that even sub-nanometre lateral resolution can be obtained by detecting the higher order non-linear dielectric constants. However, in this case the non-linear dielectric response will be related to a much thinner surface layer. Using a slightly modified probe, it is possible to measure $\varepsilon 311$ component of the dielectric tensor and thus detect the polarization component parallel to the surface potentially allowing three-dimensional reconstruction of polarization orientation .

The AFAM technique has been instrumental in evaluating the mechanical properties of ferroelectrics at the nanoscale, which is an important step forward for ferroelectric application in micro- and nanoelectrical mechanical systems. In the AFAM, the cantilever vibrates at its resonant frequency while the tip remains in contact with the sample surface. Due to the size of the SPM cantilevers, the frequencies of their vibration modes are typically in the ultrasonic range. Since, the resonance frequency in contact depends on the mechanics of the tip-sample contact, variations in the elastic properties of the sample lead to a shift in the resonance frequency and to variations in the oscillation amplitude. These changes can be used either in the spectroscopic mode for quantitative evaluation of the local contact stiffness or in the scanning mode for two-dimensional mapping.

The most important issues of PFM characterization include the PFM contrast formation, dynamic properties of the cantilever and contribution of the electromechanical response and electrostatic forces to the PFM signal, electroelastic field distribution inside the ferroelectric, local domain switching behaviour and PFM spectroscopy via local hysteresis loop acquisition elastic properties.

In general, the amplitude of the cantilever vibration in the PFM mode can be presented as $A=A_{\text {piezo }}+A_{\text {cap }}+A_{n l}$, where $A_{\text {piezo }}$ is the electromechanical response of the surface due to converse piezoelectric effect, $A_{\text {cap }}$ is the contribution due to the electrostatic force between the tip and the sample and $A_{n l}$ is the contribution due to non-local electrostatic force between the cantilever and the sample. Typically, when the domain size is much smaller than the cantilever length, non-local interaction results only in a constant background (offset) that can be easily subtracted from the PFM signal. This allows the non-local signal to be neglected for many practical cases. One more issue is that a system composed of a conducting tip in contact with the dielectric surface on a conductive substrate can be considered as a capacitor. Therefore, an external voltage $V$ applied between the tip and the counter electrode results in an additional capacitive (Maxwell) force that can be determined by the following equation: It follows that, if a combination of the dc and ac voltages, $V=V \mathrm{dc}+V \omega \cos \omega t$ ( $V \omega$ is an amplitude of an ac voltage) is applied between the tip and the counter electrode, the capacitive force will consist of a static force $F 0$, a force oscillating at a driving frequency $\omega$, as well as a force oscillating at double frequency $2 \omega$. These forces can be essential even in the contact mode and must be compared with other forces including contact force exerted by the cantilever and electromechanical force acting on the tip due to the piezoelectric effect. Applications of PFM for high-density data storage and ferroelectric lithography necessitate studies of both thermodynamics and kinetics of the switching process. The first analysis of the thermodynamics of domain switching in PFM capturing the essential features of this process including finite nucleation bias was given by Abplanalp. The closed form solution for domain switching in the point charge approximation was given by Molotskii et. al. Thermodynamic description of domain switching based on rigorously derived ferroelectroelastic fields was developed recently. Analysis of this problem was further developed by Morozovska and Eliseev. In all these papers, it was shown that the spatial extent of the field produced by the tip was confined below the tip; therefore, the switching had to be 
limited to a small volume of material. For point charge type models with charge located on or below the ferroelectric surface, the field in the vicinity of the tip was infinite and domain nucleation was induced at arbitrarily small biases. In the models that considered finite tip-surface separation or that were based on realistic tip geometry, finite nucleation bias was predicted. In the former case, using exact closed form solutions for the electroelastic fields, the domain nucleation under the PFM conditions could be described in terms of the Landau theory of phase transitions. In this case, the domain size played the role of the order parameter and the applied bias could be considered as a temperature.

Also, it was shown that for a point charge on the surface or inside the ferroelectric, domain nucleation could be considered as a second order phase transition. However, for the charge above the surface and for the realistic tip shape, the switching should be considered as a 1st order process. For the pure ferroelectric switching, the domain size was independent of the contact area and was determined solely by the tip charge or force. On the contrary, in the high order ferroelectroelastic switching the tip-surface contact contribution to the domain free energy dominated due to the much higher concentration of electroelastic and elastic fields below the tip. This analysis was very useful for determining the smallest possible size of the artificially created domains that could be produced for high-density data storage. Figure 4 illustrates domain sizes obtained using ferroelectric and ferroelectroelastic switching in PZT thin films and LiNbO3 single crystals.

\section{Ferroelectric Memory Based on Nanostructures}

In the past decades, ferroelectric materials have attracted wide attention due to their applications in nonvolatile memory devices (NVMDs) rendered by the electrically switchable spontaneous polarizations. Furthermore, the combination of ferroelectric and nanomaterials opens a new route to fabricating a nanoscale memory device with ultrahigh memory integration, which greatly eases the ever increasing scaling and economic challenges encountered in the traditional semiconductor industry. In this review, we summarize the recent development of the nonvolatile ferroelectric field effect transistor (FeFET) memory devices based on nanostructures. The operating principles of FeFET are introduced first, followed by the discussion of the real FeFET memory nanodevices based on oxide nanowires, nanoparticles, semiconductor nanotetrapods, carbon nanotubes, and grapheme. In general, ferroelectrics are dielectric crystals with the perovskite structure, whose formula is $\mathrm{ABO} 3$ with the schematic structure shown in Figure 5a The spontaneous polarization arises as the temperature sweeps due to a lattice distortion which involves the relative displacements of B4+ in each cell. These ferroelectric behaviors appear only under an inherent temperature (Curie temperature TC). As shown in Figure 1b, the similar polarization phenomenon can also be exhibited from the ferroelectric under the condition of an external electric field (E), in which the intensity of polarization (P) does not exhibit a linear response to $\mathrm{E}$ but instead shows a closed hysteretic loop. When $\mathrm{E}$ strides a particular In other words, $\mathrm{P}$ can be switched by modulating $\mathrm{E}$, and the remnant polarization $+\operatorname{Pr}$ and $-\operatorname{Pr}$ states are stored in ferroelectric memories. The bistable state of ferroelectric can be programmed as binary information ' 1 ' and ' 0 ' for NVMDs is shown in Fig 6 and Fig. 7
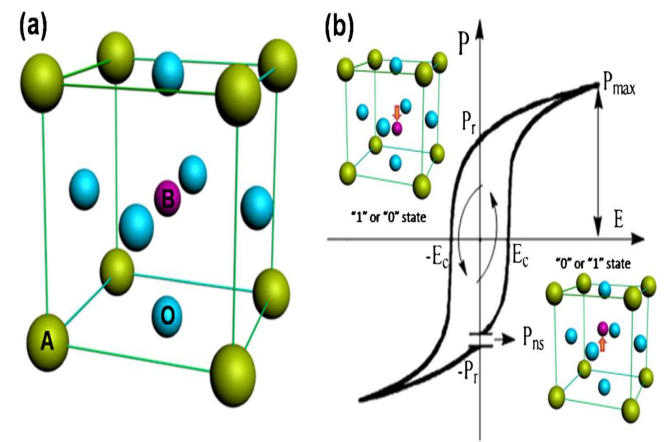

Figure 5. Schematic structure (a) and hysteresis loop curve of ferroelectric (b). In (a), the green balls represent the A2+, blue balls are O2-, and the purple one is B4+; they are located at the vertex angle, centroid, and body center, respectively. In (b), the inserts are the corresponding location of B4+ in the lattice cell, with the bistability state obviously different from that of the dynamic random access memory which needs power to maintain its state. (a)

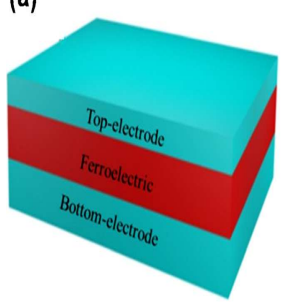

(b)

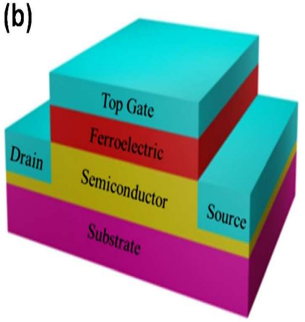

Figure 6. The current models of NVMDs. (a) The capacitor model, consisting of a thin ferroelectric film between two conductive electrodes. (b) The FeFET model, which replaces the dielectric of MOSFET with ferroelectric Oxide $N W$-based FeF
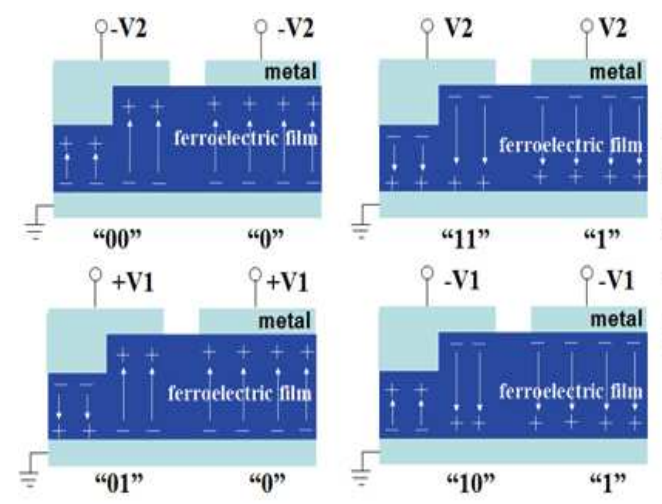

Figure 7. Sketch map of multi-bit storage operation and remnant polarization comparison between an embossed and an unembossed region. (a) Schematically illustration of multi-bit storage operation for embossed regions on a PZT film. 
Nano-embossing technique to form a stagger structure in ferroelectric lead zirconate titanate $[\mathrm{Pb}(\mathrm{Zr} 0.3, \mathrm{Ti} 0.7) \mathrm{O} 3$ $(\mathrm{PZT})]$ films and investigate the ferroelectric and electrical characterizations of the embossed and un-embossed regions, respectively, of the same films by using piezoresponse force microscopy (PFM) and Radiant Technologies Precision Material Analyzer. Attributed to the different layer thickness of the patterned ferroelectric thin film, two distinctive coercive voltages have been obtained, thereby, allowing for a single ferroelectric memory cell to contain more than one bit of data. It is widely understood that multi-bit operation could be one of the most efficient approaches to increase storage densities. In recent years, a great deal of efforts has been made on realizing multi-value storage through circuit design. One of the drawbacks is the additional budget of densities in circuit integration. There have been rarely reports on the research tackling the improvement of fabrication processes and device structures. Nano-embossing technology has emerged as a fast and cost effective technique suitable for patterning structures with feature sized progress in developing a nanoembossing technique to achieve large arrays of ferroelectric PZT cells, which have potential application in multibit storage based on ferroelectric nanostructures.

Since the principle of FeRAM is based on the polarization reversal by an externally applied electric field of metal-ferroelectric-metal capacitors, the computational ' 0 ' and ' 1 ' are represented by the nonvolatile storage of the negative or positive remnant polarization state, respectively.

\section{Conclusion}

Recent advances in fabrication and characterization of oxide ferroelectrics present both opportunities and challenges. Nanoscale ferroelectric structures have a high potential for application in electronic devices, such as high-density non-volatile memories. Rapid development of ferroelectric-based devices with reduced dimensions generated a strong need for an extensive investigation of the size effects in ferroelectric materials. Extrinsic effects on polarization stability and mechanism of switching in nanoscale volumes need to be addressed to get an insight into the performance and reliability of nanoscale devices. Scanning probe microscopy techniques provide wide opportunities for studying the physical properties and functionality of ferroelectric materials at the nanoscale level. A possibility of SPM manipulation of ferroelectric domains facilitates development of new nanofabrication approaches and devices. Clearly, the future will evidence broad application of these techniques for ferroelectrics as well as for ferroelectric-semiconductor devices. A quest for the intrinsic limit of the ferroelectric phase requires further theoretical studies that go beyond thermodynamic consideration. It is expected that ab initio calculations will play an increasingly important role in understanding the ferroelectric behaviour in one- and two dimensional nanostructures.

\section{Acknowledgements}

I would like to acknowledge Prof.C.L.R.S.V. Prasad, Principal and Professor Nagendar Parashar Director (Academic) of GMR Institute of Technology, Rajam, A.P,India for their constant encouragement to finish this work.

\section{References}

[1] Szafraniak,a) C. Harnagea,b) R. Scholz, S. Bhattacharyya,c) D. Hesse, and M. Alexe Ferroelectric epitaxial nanocrystals obtained by a self-patterning method APPLIED PHYSICS LETTERS VOLUME 83, NUMBER 1115 SEPTEMBER $20032211-2213$

[2] Ionela Vrejoiu,a_ Marin Alexe, Dietrich Hesse, and Ulrich Gösele Ferroelectric nanostructures J. Vac. Sci. Technol. B 27,1 Jan/Feb 2009 pp. 498-503.

[3] A Gruverman and A Kholkin Nanoscale ferroelectrics: processing, characterization and future trends Rep. Prog. Phys. 69 (2006) 2443-2474

[4] Xingqiang Liu, Yueli Liu, Wen Chen, Jinchai Li and Ferroelectric memory based on nanostructures Nanoscale Research Letters 2012, 7:285-290

[5] Zhenkui Shen, Zhihui Chen, Qian Lu, Zhijun Qiu, Anquan Jiang, Xinping $\mathrm{Qu}$, Yifang Chen and Ran Liu , Nano-embossing technology on ferroelectric thin film $\mathrm{Pb}(\mathrm{Zr} 0.3$,Ti0.7)O3 for multi-bit storage application Nanoscale Research Letters 2011, 6:474-479,

[6] Zhaohui Ren, Gang Xu, Xiao Wei, Yong Liu, Ge Shen, and Gaorong HanwShape Evolution of $\mathrm{Pb}$ (Zr,Ti)O3 Nanocrystals Under Hydrothermal Conditions J. Am. Ceram. Soc., 90 [8] 2645-2648 (2007)

[7] Waser R (ed) 2005 Nanoelectronics and Information Technology: Advanced Electronic Materials and Novel Devices (Berlin: Wiley-VCH)

[8] Hong S 2003 Nanoscale Phenomena in Ferroelectric Thin Films (Dordrecht: Kluwer)

[9] Alexe M and Gruverman A 2004 Nanoscale Characterization of Ferroelectric: Scanning Probe Microscopy Approach (Berlin: Springer). 